\title{
First application of carbon-based screen-printed electrodes for the voltammetric determination of the organic UV filters oxybenzone and octocrylene
}

Adrià Sunyer ${ }^{1}$, Ainoa González-Navarro ${ }^{1}$, M. Pau Serra-Roig ${ }^{2}$, Núria Serrano ${ }^{1}$, M. Silvia Díaz-Cruz ${ }^{2}$ and José Manuel Díaz-Cruz ${ }^{1,3}$ *

1 Department of Chemical Engineering and Analytical Chemistry, University of Barcelona, Martí i Franquès 1-11, 08028-Barcelona, Spain.

2 Department of Environmental Chemistry, Institute of Environmental Assessment and Water Research (IDAEA), Spanish Council of Scientific Research (CSIC), Jordi Girona 18-26, 08034Barcelona, Spain

3 Institut de Recerca de l'Aigua (IdRA), University of Barcelona.

*Corresponding author. Phone: (34) 9340217 96, e-mail: josemanuel.diaz@ub.edu

\begin{abstract}
A new voltammetric methodology is proposed for the simultaneous determination of the organic UV filters oxybenzone (benzophenone 3, BP3) and octocrylene $(\mathrm{OC}$ ) in personal care products and in wastewater extracts. It is based on previous adsorptive stripping voltammetric (AdSV) methods developed for hanging mercury drop electrodes, adapted to the special characteristics of carbon-based screen-printed electrodes. Among the carbon substrates tested, regular carbon screen-printed devices exhibited the best performance, with detection limits of 4.8 and $6.6 \mu \mathrm{mol} \mathrm{L}^{-1}$ and linearity ranges of $16-400$ and $22-400 \mu \mathrm{mol} \mathrm{L} \mathrm{L}^{-1}$ for BP3 and OC, respectively, and, as compared to mercury electrodes, with the advantages of environmental safety, easy of use, low cost and compatibility with automation and flow measurements. The methodology has been successfully tested in real samples and compared to the standard methodology by liquid chromatography - tandem mass spectrometry (LC-
\end{abstract} MS/MS).

Keywords: Personal care products; oxybenzone (BP3); octocrylene (OC); stripping voltammetry; screen-printed electrodes; LC-MS/MS. 


\section{Introduction}

Human activities are constantly increasing the number of contaminants released into the environment. Among these, ultra-violet filters (UVF) have recently emerged and are currently considered as contaminants of emerging concern (CEC). UVF are inorganic and organic chemicals used to attenuate the negative effects of sunlight exposure on both human skin and synthetic materials. Thus, they can be found not only in hygiene and personal care products such as sunscreens and cosmetics (perfumes, cosmetics, hair sprays, shampoos,...), but also in industrial products requiring protection against photodegradation (plastics, adhesives, paints, rubbers-..) [1-4]. Inorganic UVF, mainly titanium dioxide, protect the skin by reflecting and scattering UV radiation. More interesting from an analytical point of view are organic UVF, which absorb UV radiation to produce an excited state of the molecule. The excess of energy is further dissipated by emission of higher wavelength radiations (fluorescence), by photochemical processes like isomerisation or by heat release [2].

In the past few years, the increasing risk awareness about excessive sun radiation (responsible for skin cancer and photoaging) has promoted an extensive use of personal care products with high sun protection factor (SPF). In order to enhance the SPF values, several combinations of UVF are used and their total concentration in the final products increases. Considering that most hygiene and personal care products will find their way into wastewater and consequently into rivers, lakes and oceans, it is not surprising that organic UVF, as well as their photodegradates and metabolites, are frequently found in the environment [3-14]. Due to their physicochemical characteristics, most of them tend to bioaccumulate in biota. It is especially relevant the presence of UVF in dolphins [15] and its maternal transfer to foetuses [16], as well as the UVF occurrence in birds eggs [17] and in humans; breast milk, placenta, urine and semen [18-20].

UVF have shown to display estrogenic and androgenic activity in in vivo and in vitro studies [21, 22]. Oral administration of UVF to mammals during pre and postnatal life has revealed their endocrine activity, being both central nervous system and reproductive organs the main targets for the damaging effects of such activity [18]. Therefore, the occurrence of UVF in the environment constitutes a threat to natural ecosystems and an important risk to human health, which demands cost effective, fast and environmental friendly methods for the monitoring of these substances in different matrices.

Depending on the needs, the determination of UVF involves two main levels, i.e., in the consumer's products, where concentrations are high, for instance in sunscreens [23], or in 
environmental samples, where concentrations are in the low ppb or ppt level [24]. In the first case, European regulations provide a list of the UVF substances allowed in cosmetic products and their maximum concentration. There are no official methods to determine them, but the most popular technique is reverse-phase liquid chromatography with UV-vis detection [24, 25]. For environmental samples, the need of high selectivity and sensitivity promoted the application of methodologies based on reverse-phase liquid chromatography-tandem mass spectrometry (LC-MS/MS) [26] including an exhaustive sample pre-treatment, involving extraction, purification and pre-concentration.

Despite many organic UVF are electroactive, only a few electrochemical methods have been proposed for their analysis. L.H. Wang used voltammetry at mercury film electrodes to determine some UVF substances in cosmetics [27], L.F. Moreira et al. developed amperometric sensors based on polyaniline immobilised in stainless steel electrodes [28], and the group of V.S. Ferreira applied differential pulse and square wave adsorptive stripping voltammetry (AdSV) at mercury drop [29-31] and glassy carbon [32] electrodes to determine up to three sunscreen agents in commercial preparations. The last methodologies [29-32] are based on the accumulation of the analytes by adsorption on the electrode surface and the further reduction to produce a cathodic peak. They also employ methanol:water and ethanol:water mixtures as well as tensoactive substances (typically cetyltrimethylammonium bromide, CTAB) to increase the solubility of the most hydrophobic analytes (typically octocrylene). Although such electroanalytical methods, as compared to the chromatographic ones, present the inherent advantages of low cost, simplicity and portability, they have been scarcely used due to different reasons: the lack of selectivity, the poor reproducibility of solid electrodes, the restrictions in the use of mercury and, in the case of environmental samples, the low sensitivity.

But this situation could change with the recent popularisation of screen-printed electrodes (SPEs) [33-38]. These devices are obtained by printing on a plastic or ceramic support a series of inks containing the components of the working, reference and auxiliary electrode and the required connectors. Screen-printed electrodes are disposable and low-cost devices quite reproducible from one unit to the other which do not need tedious polishing and activation procedures previous to the measurement. Moreover, their design versatility and the option to use a wide variety of printing inks compositions, as well as the easy modification of their surface are important advantages of these devices. In addition, they can be easily adapted to 
flow and automated systems, and simply connected to portable instrumentation making them more suitable for in situ analysis.

In this context, the present work aims at evaluating the possibilities of voltammetry coupled to SPEs to constitute a complement to the more powerful yet expensive chromatographic-mass spectrometry methods. In the case of sunscreen products, voltammetric measurements using SPEs could determine UVF, where they are the main active component. For environmental samples, voltammetry at SPEs could be used as a screening method in a small volume of preconcentrated (e.g. by solid phase extraction) sample.

For this purpose, we selected the widespread sunscreen agent 2-hydroxy-4methoxybenzophenone, usually known as oxybenzone, benzophenone-3 or BP3, which has been extensively analysed by LC-MS/MS based methods, but also with mercury and glassy carbon electrodes [29-31]. Octocrylene (OC) was also tested as a substance considerably more hydrophobic than BP3, but with similar electrochemical properties [31, 32]. Figure 1 shows the chemical structure of both BP3 and OC molecules. As for the SPEs, two types of carbon-based electrodes have been tested: screen-printed carbon electrodes (SPCE) and carbon nanofibers modified screen-printed electrode (SPCNFE). Finally, in order to test the suitability of the developed method for the simultaneous analysis of BP3 and OC at high and low concentrations, two commercial sunscreens products and sea water samples were analysed.

\section{Materials and methods}

\subsection{Reagents}

All reagents employed, unless otherwise stated, were of analytical grade. The UV filters 2oxybenzone (BP3) with a purity of $98 \%$ and octocrylene (OC) with a purity of $97 \%$, the surfactant hexadecyltrimethylammonium bromide (CTAB) and the solvents acetonitrile ( $A C N)$, dichloromethane (DCM) and ethyl acetate (AcEt) were purchased from Sigma-Aldrich (Steinheim, Germany). Hydrochloric acid, sodium hydroxyde, formic acid, absolute ethanol and methanol were obtained from Panreac Applichem (Castellar del Vallès, Spain). The personal care product samples (a sunscreen cream and a sunscreen oil) were purchased in local markets. All solutions were prepared in purified water obtained from an Elix 3 coupled to a Milli-Q system by Millipore (Bedford, MA, USA). 


\subsection{Sample treatment}

Two commercial sunscreen products from a local market were analysed: a cream containing 5 $\%$ of BP3, according to the manufacturer, and an oil containing an unspecified amount of both BP3 and OC. Stock solutions of the samples were prepared weighting accurately $1 \mathrm{~g}$ of the product and dissolving it with methanol. In the case of the cream, the solution was filtered with a $0.45 \mu \mathrm{m}$ Whatman paper using a simple glass funnel. Then, it was made up with methanol in order to remove the insoluble excipient. From these stock solutions, dilutions with methanol or with 20:80 methanol:water mixtures were made for LC or voltammetric measurements, respectively, to get the analyte concentration within the linearity range.

$200 \mathrm{~mL}$ of the sea water samples were extracted using $500 \mathrm{mg} / 12 \mathrm{ml}$ StrataTM-X $33 \mathrm{~mm}$ polymeric reversed phase C18 cartridges Phenomenex (Torrance, CA, US). For the analytes elution, $3 \times 2.5 \mathrm{~mL}$ AcEt/DCM $(1: 1, \mathrm{v} / \mathrm{v})$ and $2 \times 1 \mathrm{~mL}$ DCM were successively used. Finally, the extracts were evaporated to dryness and reconstituted with a small volume of a suitable solvent $(0.5 \mathrm{~mL}$ of the LC-mobile phase containing the internal standards shown in Table 1 , or the measuring medium in voltammetry). Finally, $20 \mu \mathrm{l}$ of the extracts were analyzed by LC$\mathrm{MS} / \mathrm{MS}$, and $0.75 \mathrm{~mL}$ by voltammetry.

\subsection{Electrochemical analysis}

Differential pulse adsorptive stripping voltammetric (DPAdSV) and cyclic voltammetric (CV) measurements were carried out with an electrochemical system by Metrohm (Herisau, Switzerland) consisting of a $\mu$-Autolab Type III potentiostat attached to a 663 VA Stand and to a personal computer equipped with GPES 4.9 software. Screen-printed electrodes of carbon (reference DRP-110), and carbon nanofibers (reference DRP-110CNF) were purchased from Dropsens (Oviedo, Spain) and attached to the VA Stand by means of a flexible cable (reference CAC) by Dropsens. In order to improve the accuracy of the measurements, an external $\mathrm{Ag} / \mathrm{AgCl}$ reference electrode and an external platinum auxiliary electrode (both by Metrohm) were used.

For regular electrochemical measurements, synthetic and commercial sunscreens solutions containing the standards BP3 and/or OC were prepared in a 20:80 v/v methanol:water medium containing $0.1 \mathrm{~mol} \mathrm{~L}^{-1}$ of formic-formiate buffer at $\mathrm{pH} 4.0$ and, in some cases, different concentrations of CTAB. Then $25 \mathrm{~mL}$ of these solutions were placed in a glass cell (Metrohm) and were measured by DPAdSV or CV. Calibration plots were obtained by successive additions of BP3 and OC standards into a blank solution (the same above-described medium without 
analytes). The quantification in the sunscreen and water extract samples was carried by means a single determination was made for the water extract.

When not otherwise indicated, DPAdSV measurements were performed with a deposition potential of $-0.6 \mathrm{~V}$, a deposition time of $80 \mathrm{~s}$ and a differential pulse scan from $-0.7 \mathrm{~V}$ to $-1.7 \mathrm{~V}$ using pulse heights of $50 \mathrm{mV}$, pulse times of $50 \mathrm{~ms}$ and scan rates of $20 \mathrm{mV} \mathrm{s}^{-1}$. In order to remove from the electrode surface the substances produced by reduction of the analytes, a conditioning oxidative potential of $-0.2 \mathrm{~V}$ was applied for $60 \mathrm{~s}$ before each new measurement.

In the case of the surface water extract, the scarce volume available required the use of a polyethylene cell to contain a small volume of solution $(0.75 \mathrm{~mL})$ in contact with the SPE device and the reference and auxiliary electrodes also available in the same SPE unit.

\subsection{LC-MS/MS analysis}

LC-MS/MS analyses were performed in a HPLC Symbiosis ${ }^{\mathrm{TM}}$ Pico from Spark Holland (The Netherlands) attached to an hybrid quadrupole linear ion trap mass spectrometer 4000 QTRAPTM MS/MS from Applied Biosystems-Sciex (California, USA).

LC separation was achieved in a Hilar Purosher STAR HR R-18 ec $(50 \mathrm{~mm} \times 2.0 \mathrm{~mm}, 5 \mu \mathrm{m})$ LCcolumn with a precolumn of the same material. The mobile phase consisted of water and acetonitrile (ACN), both $0.1 \%$ in formic acid, with flow of $0.2 \mathrm{~mL} / \mathrm{min}$. The elution gradient started with $5 \%$ ACN, increasing to $75 \%$ in $7 \mathrm{~min}$, and up to $100 \%$ in the following $3 \mathrm{~min}$. Constant $100 \%$ organic phase was maintained for $5 \mathrm{~min}$ and was returned to the initial conditions in $2 \mathrm{~min}$. The injection volume was $20 \mu \mathrm{L}$.

MS/MS detection was operated under positive electrospray ionisation (ESI+) and selected reactions monitoring (SRM) modes with optimum conditions as follows: voltage of the capillary, $5000 \mathrm{~V}$; source temperature, $700{ }^{\circ} \mathrm{C}$, curtain gas, $30 \mathrm{psi}$; ion gas source $1.50 \mathrm{psi}$; gas from the source of ions $2.60 \mathrm{psi}$; input potential, $10 \mathrm{~V}$. The two most characteristic fragments of the precursor molecular ion $[\mathrm{M}+\mathrm{H}]^{+}$were monitored for each analyte. The most abundant and the second most abundant transitions were used for quantification and confirmation, respectively. The precursor-product ion transitions for the target analytes and the isotopically labelled standards (IS) used for the quantification (isotope dilution method) are listed in Table 1. Under these optimized conditions, the linearity and sensitivity, expressed as limits of detection (LOD) and quantification (LOQ), were: $2 \cdot 10^{-5}-2 \mu \mathrm{mol} \mathrm{L}^{-1}, 4 \cdot 10^{-6}$ and $2 \cdot 10^{-5} \mu \mathrm{mol} \mathrm{L}^{-1}$ for BP3, and $4 \cdot 10^{-5}-2 \mu \mathrm{mol} \mathrm{L}^{-1}, 9 \cdot 10^{-6}$ and $4 \cdot 10^{-5} \mu \mathrm{mol} \mathrm{L}^{-1}$ for OC. 


\section{Results and Discussion}

\subsection{Preliminary study by cyclic voltammetry}

In order to investigate the electrochemical behaviour of BP3 and OC at the SPEs considered, a preliminary study was performed by $\mathrm{CV}$ using the simplest and cheapest device, i.e., a carbon screen-printed electrode (SPCE). During this study, it was found that BP3 could be easily dissolved in methanol:water mixture 20:80 in the presence of the formic-formiate buffer at $\mathrm{pH}$ 4.0. In contrast, $O C$ also required a certain amount of CTAB to prevent precipitation $\left(1.2 \cdot 10^{-3}\right.$ $\mathrm{mol} \mathrm{L}^{-1}$ in these experiments). Figure 2 shows the signals measured for both BP3 (Figure 2a) and OC (Figure $2 \mathrm{~b}$ ) at different scan rates using a SPCE. In both cases a single and broad cathodic peak is observed in the forward scan indicating the reduction of the analytes. This happens at more negative potentials for BP3 ( $c a .-1.2 \mathrm{~V}$ at the lowest rates) as compared to OC (ca. -1.1 V). The absence of the corresponding anodic peak in the backward scan and the dramatic shift of the cathodic peak towards negative potentials at increasing scan rates (more than $200 \mathrm{mV}$ ) evidence the irreversible character of the reduction of BP3 and OC at the SPCE, as it had been previously reported in the case of mercury electrodes [29-31].

This preliminary study also showed that, as previously observed working with mercury electrodes, the products of the reduction caused fouling of the electrode surface, resulting in a progressive decrease of the signals when repeated measurements were carried out in the same solution. This problem was successfully solved by applying a conditioning oxidative potential of $-0.2 \mathrm{~V}$ during $60 \mathrm{~s}$ before registering every new voltammogram. In this way, currents were stable along repeated measurements.

\subsection{Optimization of DPAdSV measurements}

Although the deposition potential did not affect significantly the magnitude of the reduction peak, the best conditions for both BP3 and OC appeared to be close to $-0.6 \mathrm{~V}$, which was selected as the optimal value. As an example, Figure 3 compares the DPAdSV signals for BP3 obtained at different deposition potentials.

Concerning to the deposition time, experiments carried out with $\mathrm{OC}$ at concentration levels of the order of $10^{-5} \mathrm{~mol} \mathrm{~L}^{-1}$ showed that $80 \mathrm{~s}$ was a convenient value, since at higher times stripping currents increased less than expected as a consequence of the saturation of the electrode (Figure 4). Although BP3 could be analysed with quite longer deposition times without significant saturation, the value of $80 \mathrm{~s}$ was considered a good compromise for the simultaneous determination of both analytes. 
As for the optimal amount of CTAB, it was observed that increasing concentrations of $O C$ optimisation, it was found that a CTAB concentration of $6 \cdot 10^{-3} \mathrm{~mol} \mathrm{~L}^{-1}$ allowed linearity ranges up to $3 \cdot 10^{-4} \mathrm{~mol} \mathrm{~L}^{-1}$ of $\mathrm{OC}$ without noticeable problems such as analyte precipitation or electrode fouling.

\subsection{Comparison of different carbon-based screen-printed electrodes}

In order to select the most convenient SPE for the simultaneous DPAdSV determination of BP3 and OC, calibration graphs were obtained separately for both compounds by using SPCE (the cheapest option) and SPCNFE (as the presence of such carbon nanofibers had provided especially good results in previous works by our group [39, 40]). The experimental conditions used were these stablished in the preliminary study (deposition potential of $-0.6 \mathrm{~V}$, deposition time of $80 \mathrm{~s}$ and CTAB concentration of $6 \cdot 10^{-3} \mathrm{~mol} \mathrm{~L}^{-1}$ in the case of $\mathrm{OC}$ ). Table 2 summarises the performance of the developed methodology using SPCE and SPCNFE devices. Good linear responses of peak area vs. BP3 and OC concentration have been respectively achieved using both SPCE and SPCNFE. Regarding sensitivities, estimated as the slope value of the calibration curves of BP3 and OC, respectively, it can be stated that the sensitivity for BP3 at SPCNFE was somewhat higher than that using a SPCE as it was expected considering the much larger effective surface area that present the SPCNFE in comparison to the conventional unmodified SPCE. In contrast, for the determination of OC at SPCNFE, sensitivity was practically the same as that achieved with SPCE, likely due to the presence of CTAB as a surfactant. Detection limits (LOD) and quantification limits (LOQ) have been computed as three times or ten times the standard deviation of the calibration line divided by the slope, respectively. As it can be seen in Table 2, SPCE devices provided the lowest LODs and the widest linear ranges. Taking into account previous results $[39,40]$, it is a bit surprising the poorer performance of SPCNFE devices, which present a much higher specific surface than regular SPCE devices. This could be due to the need for electrochemical removal of the products of UVF reduction to prevent electrode fouling and current decay. Maybe the cleaning of the nanostructured surface of SPCNFE units is not as effective as in the case of SPCE units. Therefore, not only at the view of the above results, but also considering that SPCE are the cheapest devices, they were selected to perform the further analyses. Moreover, additional studies of repeatability and reproducibility were carried out, consisting of 7 consecutive measurements with the same SPCE unit in three different days (of course by using a new unit every day). The study yielded a 
RSD \% of ca. $2 \%$ using the same unit along the same day and $1 \%$ when comparing the averages of the three different days, thus confirming an excellent repeatability and reproducibility of the voltammetric method using the SPCE units.

The analytical performance of SPCE is likely not as good of that of mercury electrodes, with LODs of the order of $n m o l L^{-1}$ [29-31], but in contrast SPCE devices are less toxic, more reproducible, and suitable for in situ and flow measurements. Moreover, it should be pointed out $t$ that they do not require any pre-polishing, are disposable, commercially available, and of low-cost.

\subsection{Simultaneous determination of BP3 and OC by DPAdSV at SPCE}

Once the main experimental conditions were studied for both analytes individually, the simultaneous determination of BP3 and OC with SPCE was considered. In an initial experiment in the tested condition were as follows: $20: 80 \mathrm{v} / \mathrm{v}$ methanol:water medium with CTAB $6 \cdot 10^{-3}$ mol L ${ }^{-1}$ and formic / formiate buffer $0.1 \mathrm{~mol} \mathrm{~L}^{-1}$ at $\mathrm{pH} 4.0$, a deposition potential of $-0.6 \mathrm{~V}$ and deposition time of $80 \mathrm{~s}$. Figure 5 and Table 3 summarise the results obtained. As compared to the individual measurements of BP3 and OC, the detection and quantification limits achieved were slightly higher and the sensitivities were somewhat lower than those afforded in the individual analyses, likely as a consequence of the interactions between analytes and their competition to be adsorbed and accumulated onto the electrode surface. Anyway, these parameters were still quite good.In the view of the results (Table 3), it could be stated that the saturation of the electrode was not an issue, being possible to analyse relatively high concentrations of both compounds simultaneously. Indeed, linearity was maintained up to concentrations of $400 \mu \mathrm{mol} \mathrm{L}^{-1}$ (Figure $5 \mathrm{~b}$ ), slightly higher than in individual measurements. At increased concentrations, signals became broader and their areas started to deviate from linearity (data not shown). These results confirmed the applicability of the SPCE electrodes for the simultaneous determination by DPAdSV of low concentration levels of BP3 and OC in both personal care products and environmental samples.

\subsection{Application to the analysis of real samples}

The applicability of the developed SPCE-based method to two types of real samples was assessed, on the one hand, two commercial sunscreens (a cream and an oil) with high concentrations of the target UVF and, on the other hand, two sea water samples from a beach.

Figure 6 shows the characteristic signals obtained in the analysis of the sunscreen commercial products (an oil sample in this case) and Table 4 compares the concentrations determined by 
DPAdSV and LC-MS/MS with the concentrations declared by the manufacturer. It should be mentioned that the same device was used for the measurements of a complete replicate. A very good agreement was found between the BP3 concentrations determined by the proposed DPAdSV method using SPCE and the LC-MS/MS method. The OC results are not so similar, but still reliable as a screening tool considering the higher standard deviation of DPAdSV as compared to that of LC-MS/MS. This better performance of the method for BP3 as compared to $O C$ could be related to the quite hydrophobic character of the latter, which has a greater tendency to precipitate than BP3, even in the presence of CTAB. It must be pointed out that both personal care products analysed contained many additives and additional UV filters that, according to the results, were not interfering the voltammetric determination.

As regards the water extracts, BP3 could be detected by DPAdSV at SPCE in one sample at $7.46 \cdot 10^{-5} \mathrm{~mol} \mathrm{~L}^{-1}$ in close agreement with that determined by LC-MS/MS $\left(7.49 \cdot 10^{-5} \mathrm{~mol} \mathrm{~L}^{-1}\right)$, documenting the suitability of SCPE for the determination of the target UVF in environmental samples, where many potentially interfering substances are present. Unfortunately, the scarce extract volume available prevented us to perform duplicate analyses. OC could not be determined in any of the water extracts considered by neither of the two techniques, being not present or below the LODs.

\section{Conclusions}

As far as we know, the proposed DPAdSV method is the first successful approach for the determination of BP3 and OC using screen-printed electrodes with the additional advantages of such devices, i.e. low toxicity, good reproducibility, disposable character and low-cost. Furthermore, the proposed electrode can be used for a large set of measurements without degradation or loss of sensitivity. To the light of these results it can be concluded that DPAdSV at SPCE constitute a fast, simple and cost-effective screening tool for the determination of the organic UV filters BP3 and OC in different types of samples and, hence, a good and cheaper complement for the more accurate yet expensive LC-MS/MS methodology usually employed for their analysis. The proposed method is sensitive enough to directly determine these compounds in personal care products, but requires a pre-concentration step for the analysis of environmental samples, where these compounds are present at trace level. Therefore, for environmental analytical applications of DPAdSV at SPCE further research is required to develop procedures allowing a more extensive pre-concentration of the analytes on the electrode surface. 


\section{Acknowledgements}

The authors acknowledge the support of Generalitat de Catalunya, Spain (Project 2017SGR311), the Water Research Institute of the Universitat de Barcelona (IdRA) and the Spanish Ministry of Science, Innovation and Universities through the Project ROUSSEAU (CTM2017-89767-C3-1-R).

\section{References}

[1] F.P. Gasparro, M. Mitchnick, J.F. Nash, A review of sunscreen safety and efficacy, Photochem. Photobiol., 68 (1998) 243-256. https://doi.org/10.1111/j.1751-1097.1998.tb09677.x.

[2] N. Serpone, D. Dondi, A. Albini, Inorganic and organic UV filters: Their role and efficacy in sunscreens and suncare products, Inorg. Chim. Acta, 360 (2007) 794-802. https://doi.org/10.1016/j.ica.2005.12.057.

[3] D.L. Giokas, A. Salvador, A. Chisvert, UV filters: from sunscreens to human body and the environment, Trends Anal. Chem., 26 (2007) 360-374. https://doi.org/10.1016/j.trac.2007.02.012.

[4] M.S. Díaz-Cruz, M. Llorca, D. Barceló, Organic UV filters and their photodegradates, metabolites and disinfection by-products in the aquatic environment, Trends Anal. Chem., 27 (2008) 873-887. https://doi.org/10.1016/j.trac.2008.08.012.

[5] M.E. Balmer, H.R. Buser, M.D. Müller, T. Poiger, Occurrence of some organic UV filters in wastewater, in surface waters, and in fish from Swiss lakes, Environ. Sci. Technol., 39 (2005) 953-962. https://doi.org/10.1021/es040055r.

[6] H.R. Buser, M.E. Balmer, P. Schmid, M. Kohler, Occurrence of UV filters 4methylbenzylidene camphor and octocrylene in fish from various Swiss rivers with inputs from wastewater treatment plants, Environ. Sci. Technol., 40 (2006) 1427-1431. https://doi.org/10.1021/es052088s

[7] W. Li, Y. Ma, C. Guo, W. Hu, K. Liu, Y. Wang, T. Zhu, Occurrence and behavior of four of the most used sunscreen UV filters in a wastewater reclamation plant, Water Res., 41 (2007) 3506-3512. https://doi.org/10.1016/j.watres.2007.05.039.

[8] P. Cuderman, E. Heath, Determination of UV filters and antimicrobial agents in environmental water samples, Anal. Bioanal. Chem., 387 (2007) 1343-1350. https://doi.org/10.1007/s00216-006-0927-y.

[9] J.M. Brausch, G.M. Rand, A review of personal care products in the aquatic environment: environmental concentrations and toxicity, Chemosphere, 82 (2011) 15181532. https://doi.org/10.1016/j.chemosphere.2010.11.018.

[10] P. Gago-Ferrero, M.S. Díaz-Cruz, D. Barceló, Occurrence of multiclass UV filters in treated sewage sludge from wastewater treatment plants. Chemosphere, 84 (2011) 1158-1165. https://doi.org/10.1016/j.chemosphere.2011.04.003. 
[11] P. Gago-Ferrero, M.S. Diaz-Cruz, D. Barceló, An overview of UV-absorbing compounds (organic UV filters) in aquatic biota, Anal. Bioanal. Chem., 404 (2012) 2597-2610. https://doi.org/10.1007/s00216-012-6067-7.

[12] E. Magi, C. Scapolla, M. Di Carro, P. Rivaro, K.T.N. Nguyen, Emerging pollutants in aquatic environments: monitoring of UV filters in urban wastewater treatment plants. Anal. Methods, 5 (2013) 428-433. https://doi.org/10.1039/C2AY26163D.

[13] A. Jurado, P. Gago-Ferrero, E. Vàzquez-Suñé, J. Carrera, E. Pujades, M.S. Díaz-Cruz, D. Barceló, Urban groundwater contamination by residues of UV filters, Haz. Mat., 271 (2014) 141-149. https://doi.org/10.1016/j.jhazmat.2014.01.036.

[14] M.M. Tsui, H.W. Leung, T.C. Wai, N. Yamashita, S. Taniyasu, W. Liu, P.K.S Lam, M.B. Murphy, Occurrence, distribution and ecological risk assessment of multiple classes of UV filters in surface waters from different countries, Water Res., 67 (2014) 55-65. https://doi.org/10.1016/j.watres.2014.09.013.

[15] P. Gago-Ferrero, M.B. Alonso, C.P. Bertozzi, J. Marigo, L. Barbosa, M. Cremer, E.R. Secchi, A. Azevedo, J. Lailson-Brito Jr., J.P.M. Torres, O. Malm, E. Eljarrat, M.S. DíazCruz, D. Barceló, First determination of UV filters in marine mammals. Octocrylene levels in Franciscana dolphins, Environ. Sci. Technol., 47 (2013) 5619-5625. https://doi.org/10.1021/es400675y.

[16] M.B. Alonso, M.L. Feo, C. Corcellas, P. Gago-Ferrero, C.P. Bertozzi, J. Marigo, L. Flach, A.C.O. Meirelles, V.L. Carvalho, A.F. Azevedo, J.P.M. Torres, J. Lailson-Brito Jr., O. Malm, M.S. Díaz-Cruz, E. Eljarrat, D. Barceló, Toxic heritage: Maternal transfer of pyrethroid insecticides and sunscreen agents in dolphins from Brazil, Environ. Pollut., 207 (2015) 391-402. https://doi.org/10.1016/j.envpol.2015.09.039.

[17] D. Molins-Delgado, M. Máñez, A. Andreu, F. Hiraldo, E. Eljarrat, D. Barceló, M.S. DíazCruz, A potential new threat to wild life: Presence of UV filters in bird eggs from a preserved area, Environ. Sci. Technol., 51 (2017) 10983-10990.

https://doi.org/10.1021/acs.est.7b03300.

[18] M. Schlumpf, P. Schmid, S. Durrer, M. Conscience, K. Maerkel, M. Henseler, M. Gruetter, I. Herzog, S. Reolon, R. Ceccatelli, O. Faass, E. Stutz, H. Jarry, W. Wuttke, W. Lichtensteiger,. Endocrine activity and developmental toxicity of cosmetic UV filters - an update. Toxicology, 205 (2004) 113-122. https://doi.org/10.1016/j.tox.2004.06.043.

[19] I. Jiménez-Díaz, F. Vela-Soria, A. Zafra-Gómez, A. Navalón, O. Ballesteros, N. Navea, M.F. Fernández, N. Olea, J.L. Vílchez, A new liquid chromatography-tandem mass spectrometry method for determination of parabens in human placental tissue samples, Talanta, 84 (2011) 702-709. https://doi.org/10.1016/j.talanta.2011.01.075.

[20] Z. León, A. Chisvert, I. Tarazona, A. Salvador, Solid-phase extraction liquid chromatography-tandem mass spectrometry analytical method for the determination of 2-hydroxy-4-methoxybenzophenone and its metabolites in both human urine and semen, Anal. Bioanal. Chem., 398 (2010) 831-843. https://doi.org/10.1007/s00216-0103947-6.

[21] R.H. Schreurs, E. Sonneveld, J.H. Jansen, W. Seinen, B. van der Burg, Interaction of polycyclic musks and UV filters with the estrogen receptor (ER), androgen receptor (AR), 
and progesterone receptor (PR) in reporter gene bioassays. Toxicol. Sci., 83 (2004) 264272. https://doi.org/10.1093/toxsci/kfi035.

[22] M.S. Díaz-Cruz, D. Barceló, Chemical analysis and ecotoxicological effects of organic UVabsorbing compounds in aquatic ecosystems. Trends Anal. Chem., 28 (2009) 708-717. https://doi.org/10.1016/j.trac.2009.03.010.

[23] A. Salvador, A. Chisvert, Sunscreen analysis: a critical survey on UV filters determination, Anal. Chim. Acta, 537 (2005) 1-14. https://doi.org/10.1016/j.aca.2005.01.055.

[24] S. Ramos, V. Homem, A. Alves, L. Santos, Advances in analytical methods and occurrence of organic UV-filters in the environment-a review. Sci. Tot. Environ., 526 (2015) 278-311. https://doi.org/10.1016/j.scitotenv.2015.04.055.

[25] A. Salvador, A. Chisvert, An environmentally friendly ("green") reversed-phase liquid chromatography method for UV filters determination in cosmetics, Anal. Chim. Acta, 537 (2005) 15-24. https://doi.org/10.1016/j.aca.2004.12.047.

[26] P. Gago-Ferrero, M.S. Díaz-Cruz, D. Barceló, Liquid chromatography-tandem mass spectrometry for the multi-residue analysis of organic UV filters and their transformation products in the aquatic environment, Anal. Methods, 5 (2013) 355-366. https://doi.org/10.1039/C2AY26115D.

[27] L.H. Wang, Voltammetric behavior of sunscreen agents at mercury film electrode. Electroanalysis, 14 (2002) 773-781. https://doi.org/10.1002/1521-4109(200206)14:11<773::AID-ELAN773>3.0.CO;2-A

[28] L.F. Moreira, M.R. de Vasconcelos Lanza, A.A. Tanaka, M.D.P.T. Sotomayor, Selective UVfilter detection with sensors based on stainless steel electrodes modified with polyaniline doped with metal tetrasulfonated phthalocyanine films, Analyst, 134 (2009) 1453-1461. https://doi.org/10.1039/B902273B

[29] A.P. da Silva, M.A.G. Trindade, V.S. Ferreira, Polarographic determination of sunscreen agents in cosmetic products in micellar media, Talanta, 68 (2006) 679-685. https://doi.org/10.1016/j.talanta.2005.05.007

[30] J.C. Cardoso, B.M.L. Armondes, J.B. Galindo Jr., V.S. Ferreira, Simultaneous electrochemical determination of three sunscreens using cetyltrimethylammonium bromide, Colloid. Surface. B, 63 (2008) 34-40. https://doi.org/10.1016/j.colsurfb.2007.11.001.

[31] V.S. Ferreira, J.B.G. Júnior, C.M.S.C. Oliveira, R.M. Takeuchi, A.L. Santos, M.A.G. Trindade, Voltammetric analysis of sun-block preparations containing octocrylene and its association with 2-Hydroxy-4-methoxybenzophenone and octyl methoxycinnamate. Microchem. J., 106 (2013) 378-383. https://doi.org/10.1016/j.microc.2012.10.002.

[32] J.B.G. Júnior, T.A. Araujo, M.A.G. Trindade, V.S. Ferreira, Electroanalytical determination of the sunscreen agent octocrylene in cosmetic products, Int. J. Cosmetic Sci., 34 (2012) 91-96. https://doi.org/10.1111/j.1468-2494.2011.00686.x.

[33] O.D. Renedo, M.A. Alonso-Lomillo, M.A. Martínez, Recent developments in the field of screen-printed electrodes and their related applications, Talanta, 73 (2007) 202-219. https://doi.org/10.1016/j.talanta.2007.03.050 
[34] M. Li, Y.T. Li, D.W. Li, Y.T. Long, Recent developments and applications of screen-printed electrodes in environmental assays-A review, Anal. Chim. Acta, 734 (2012) 31-44. https://doi.org/10.1016/j.aca.2012.05.018

[35] Z. Taleat, A. Khoshroo, M. Mazloum-Ardakani, Screen-printed electrodes for biosensing: a review (2008-2013), Microchim. Acta, 181 (2014) 865-891. https://doi.org/10.1007/s00604-014-1181-1

[36] K.C. Honeychurch, J.P. Hart, Screen-printed electrochemical sensors for monitoring metal pollutants, Trends Anal. Chem., 22 (2003) 456-469. https://doi.org/10.1016/S0165-9936(03)00703-9.

[37] J. Barton, M.B.G. García, D.H. Santos, P. Fanjul-Bolado, A. Ribotti, M. McCaul, D. Diamond, P. Magni, Screen-printed electrodes for environmental monitoring of heavy metal ions: A review, Microchim. Acta, 183 (2016) 503-517. https://doi.org/10.1007/s00604-015-1651-0

[38] C. Ariño, N. Serrano, J.M. Díaz-Cruz, M. Esteban, Voltammetric determination of metal ions beyond mercury electrodes. A review, Anal. Chim. Acta, 990 (2017) 11-53. https://doi.org/10.1016/j.aca.2017.07.069.

[39] C. Pérez-Ràfols, N. Serrano, J.M. Díaz-Cruz, C. Ariño, M. Esteban, Glutathione modified screen-printed carbon nanofiber electrode for the voltammetric determination of metal ions in natural samples, Talanta, 155 (2016) 8-13. https://doi.org/10.1016/j.talanta.2016.04.011.

[40] C. Pérez-Ràfols, N. Serrano, J.M. Díaz-Cruz, C. Ariño, M. Esteban, New approaches to antimony film screen-printed electrodes using carbon-based nanomaterials substrates, Anal. Chim. Acta, 916 (2016) 17-23. https://doi.org/10.1016/j.aca.2016.03.003. 


\section{Figure Captions}

\section{Figure 1}

Chemical structures of BP3 and OC.

\section{Figure 2}

Cyclic voltammograms measured with a SPCE in solutions $110^{-4} \mathrm{~mol} \mathrm{~L}^{-1}$ of BP3 (a) and $4 \cdot 10^{-5}$ $\mathrm{mol} \mathrm{L}^{-1}$ of OC (b) in a 20:80 v/v methanol:water medium containing formic / formiate buffer $0.1 \mathrm{~mol} \mathrm{~L}^{-1}$ at $\mathrm{pH}$ 4.0. The OC solution also contained CTAB $1.2 \cdot 10^{-3} \mathrm{~mol} \mathrm{~L}^{-1}$. Scan rates applied were 10 (1), 20 (2), 50 (3), 100 (4) and 200 (5) $\mathrm{mV} \mathrm{s}^{-1}$. Arrows indicate the direction of forward (cathodic) and backward (anodic) scans.

\section{Figure 3}

DPAdS voltammograms measured at different deposition potentials with a SPCE in a solution $1 \cdot 10^{-5} \mathrm{~mol} \mathrm{~L}^{-1}$ of BP3 in a 20:80 v/v methanol:water medium containing a formic / formiate buffer $0.1 \mathrm{~mol} \mathrm{~L}^{-1}$ at $\mathrm{pH}$ 4.0. The signals have been moved along the $y$ axis for the sake of a better visualization.

\section{Figure 4}

Peak areas of the DPAdSV signals measured with a SPCE for a $2 \cdot 10^{-5} \mathrm{~mol} \mathrm{~L}^{-1}$ OCR solution in methanol:water 20:80 medium containing $1.2 \cdot 10^{-2} \mathrm{~mol} \mathrm{~L}^{-1}$ of CTAB and formic / formiate buffer $0.1 \mathrm{~mol} \mathrm{~L}^{-1}$ at $\mathrm{pH} 4.0$ as a function of the deposition time $\left(t_{d}\right)$. Dashed line has been computed using the points from $t_{d}=0 \mathrm{~s}$ to $t_{d}=80 \mathrm{~s}$. The corresponding equation and determination coefficient $R^{2}$ are shown in the graph.

\section{Figure 5}

Simultaneous DPAdSV measurement of BP3 and OC by using a SPCE in a 20:80 v/v methanol:water medium containing a formic / formiate buffer $0.1 \mathrm{~mol} \mathrm{~L}^{-1}$ at $\mathrm{pH} 4.0$ and CTAB $6 \cdot 10^{-3} \mathrm{~mol} \mathrm{~L}^{-1}$. A deposition potential of $-0.6 \mathrm{~V}$ and a deposition time of $80 \mathrm{~s}$ were applied. The figure shows a) the voltammograms measured in solutions containing concentrations of both $\mathrm{BP} 3$ and $\mathrm{OC}$ in the range $10-400 \mu \mathrm{mol} \mathrm{L}^{-1}$; and b) the corresponding calibration lines.

\section{Figure 6}

DPAdSV analysis of a commercial sunscreen containing BP3 and OC with a SPCE and using the standard addition method. The experimental conditions were the same as in Figure 6 . It is shown a) the voltammograms measured for the sample before and after four consecutive standard additions of both analytes in the range $10-40 \mu \mathrm{mol} \mathrm{L}^{-1}$; and b) the corresponding calibration lines. 


\section{Table 1}

Selected precursor-product ions transitions monitored in the LC-MS/MS method applied in this study. DP: Declustering potential; CE: Collision energy; CXP: Cell exit potential; IS: Internal standard

\begin{tabular}{|c|c|c|c|c|c|c|c|}
\hline Compound & Transition & Precursor & Product & DP (V) & CE (V) & CxP (V) & IS \\
\hline \multirow{2}{*}{ BP3 } & 1st & 229 & 151 & 40 & 25 & 12 & BP3-d5 \\
\cline { 2 - 8 } & 2nd & 229 & 105 & 40 & 27 & 16 & BP3-d5 \\
\hline OC & 1 st & 362 & 250 & 71 & 15 & 20 & 4 MBC-d4 \\
\cline { 2 - 8 } & 2 2nd & 362 & 232 & 71 & 27 & 12 & 4 MBC-d4 \\
\hline BP3-d5 & - & 234 & 151 & 36 & 27 & 12 & - \\
\hline 4MBC-d4 & - & 259 & 216 & 76 & 27 & 14 & - \\
\hline
\end{tabular}


Table 2

1

Comparison of the figures of merit achieved in the individual determination of BP3 and OC by DPAdSV using SPCE and SPCNFE devices. Measurements were carried out in a 20:80 v/v methanol:water medium containing formic / formiate buffer $0.1 \mathrm{~mol} \mathrm{~L}^{-1}$ at $\mathrm{pH} 4.0$ and, in the case of OC, also CTAB $6 \cdot 10^{-3} \mathrm{~mol} \mathrm{~L}^{-1}$.

\begin{tabular}{|c|l|l|l|l|l|l|}
\hline Compound & Device & $\begin{array}{l}\text { Sensitivity } \\
\left(\mathrm{AVmol}^{-1} \mathrm{~L}\right)\end{array}$ & $\mathrm{R}^{2}$ & $\begin{array}{l}\text { LOD } \\
\left(\mu \mathrm{mol} \mathrm{L}^{-1}\right)\end{array}$ & $\begin{array}{l}\text { LOQ } \\
\left(\mu \mathrm{mol} \mathrm{L}^{-1}\right)\end{array}$ & $\begin{array}{l}\text { Linear range } \\
\left(\mu \mathrm{mol} \mathrm{L}^{-1}\right)\end{array}$ \\
\hline \multirow{2}{*}{ BP3 } & SPCE & $1.4 \cdot 10^{-3}$ & 0.998 & 1.9 & 6 & $6-200$ \\
\cline { 2 - 7 } & SPCNFE & $2.4 \cdot 10^{-3}$ & 0.997 & 2.4 & 8 & $8-200$ \\
\hline \multirow{2}{*}{ OC } & SPCE & $1.6 \cdot 10^{-3}$ & 0.990 & 4.1 & 13 & $11-300$ \\
\cline { 2 - 7 } & SPCNFE & $1.6 \cdot 10^{-3}$ & 0.996 & 6.2 & 21 & $47-250$ \\
\hline
\end{tabular}


Table 3

1

Figures of merit achieved in the simultaneous determination of BP3 and OC by DPAdSV using SPCE devices. Measurements were carried out in a 20:80 v/v methanol:water medium containing formic / formiate buffer $0.1 \mathrm{~mol} \mathrm{~L}^{-1}$ at $\mathrm{pH} 4.0$ and CTAB $6 \cdot 10^{-3} \mathrm{~mol} \mathrm{~L}^{-1}$.

\begin{tabular}{|c|l|l|l|l|l|}
\hline Compound & $\begin{array}{l}\text { Sensitivity } \\
\left(\mathrm{AVmol}^{-1} \mathrm{~L}\right)\end{array}$ & $\mathrm{R}^{2}$ & $\begin{array}{l}\text { LOD } \\
\left(\mu \mathrm{mol} \mathrm{L}^{-1}\right)\end{array}$ & $\begin{array}{l}\mathrm{LOQ} \\
\left(\mu \mathrm{mol} \mathrm{L}^{-1}\right)\end{array}$ & $\begin{array}{l}\text { Linear range } \\
(\mu \mathrm{mol} \mathrm{L})\end{array}$ \\
\hline BP3 & $1.2 \cdot 10^{-3}$ & 0.999 & 4.8 & 16 & $16-400$ \\
\hline OC & $1.1 \cdot 10^{-3}$ & 0.999 & 6.6 & 22 & $22-400$ \\
\hline
\end{tabular}


Table 4

1

Comparison of the concentrations of BP3 and OC $(\% \mathrm{w} / \mathrm{w})$ determined with the application of the proposed DPAdSV using SPCE and the existing LC-MS/MS methods in the analysis of real sunscreen samples. Standard deviations are indicated with parenthesis.

\begin{tabular}{|l|l|l|l|l|l|}
\hline Sample & Analyte & $\begin{array}{l}\text { Concentration } \\
\text { (manufacturer) }\end{array}$ & $\begin{array}{l}\text { Concentration } \\
\text { (LC-MS/MS) }\end{array}$ & $\begin{array}{l}\text { Concentration } \\
\text { (DPAdSV with } \\
\text { SPCE) }\end{array}$ & $\begin{array}{l}\% \text { Error } \\
\text { SV vs. LC }\end{array}$ \\
\hline Sunscreen cream & BP3 & $5 \%$ & $4.15(0.05) \%$ & $4.1(0.1) \%$ & $1.2 \%$ \\
\hline \multirow{2}{*}{ Sunscreen oil } & BP3 & - & $4.96(0.03) \%$ & $5.02(0.08) \%$ & $1.2 \%$ \\
\cline { 2 - 7 } & OC & - & $3.56(0.01) \%$ & $4.2(0.6) \%$ & $18.3 \%$ \\
\hline
\end{tabular}


Click here to download high resolution image<smiles>COc1ccc(C(=O)c2ccccc2)c(O)c1</smiles><smiles>CCCCC(CC)COC(=O)C(C#N)=C(c1ccccc1)c1ccccc1</smiles> 
Click here to download high resolution image

a)

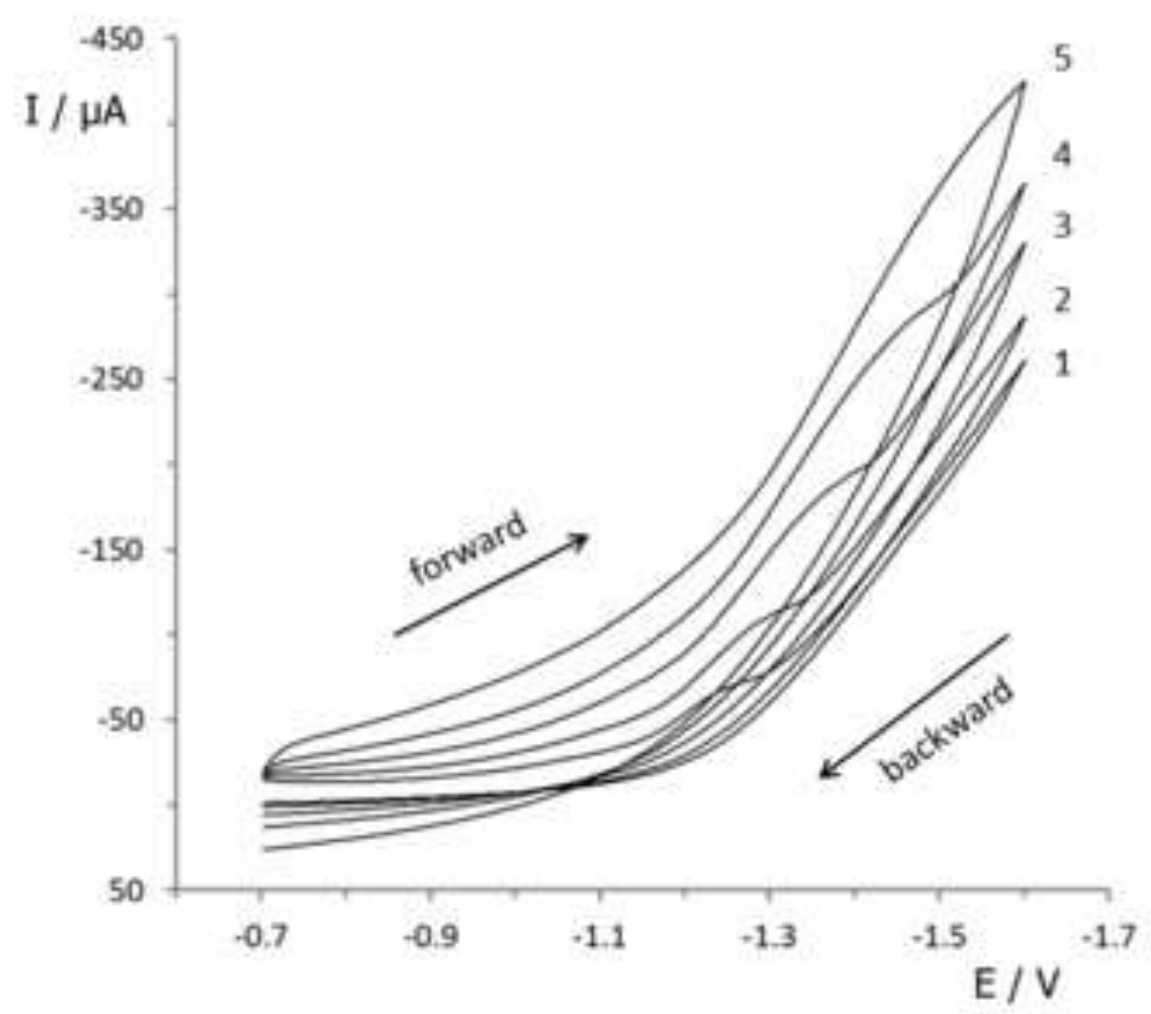

b)

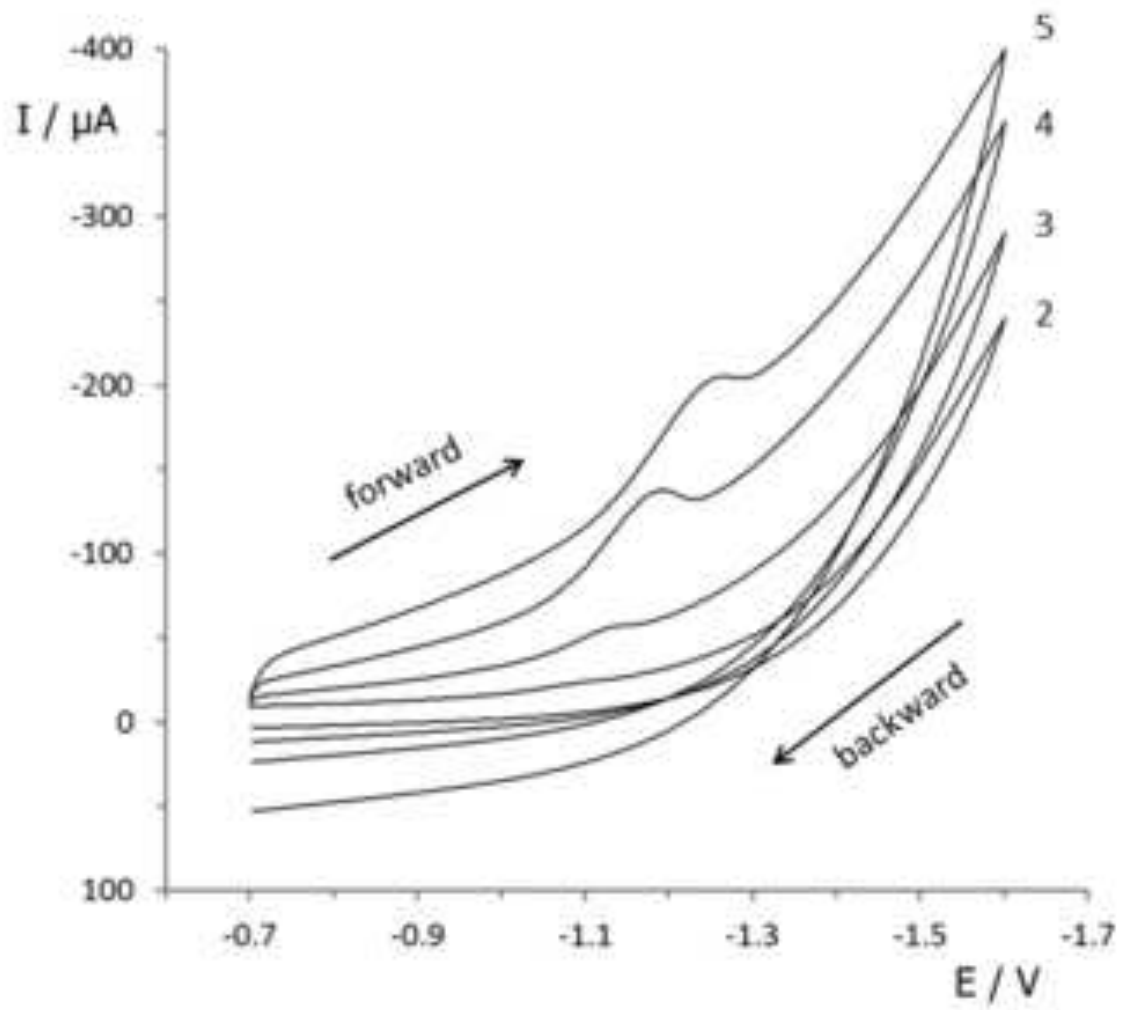


Click here to download high resolution image

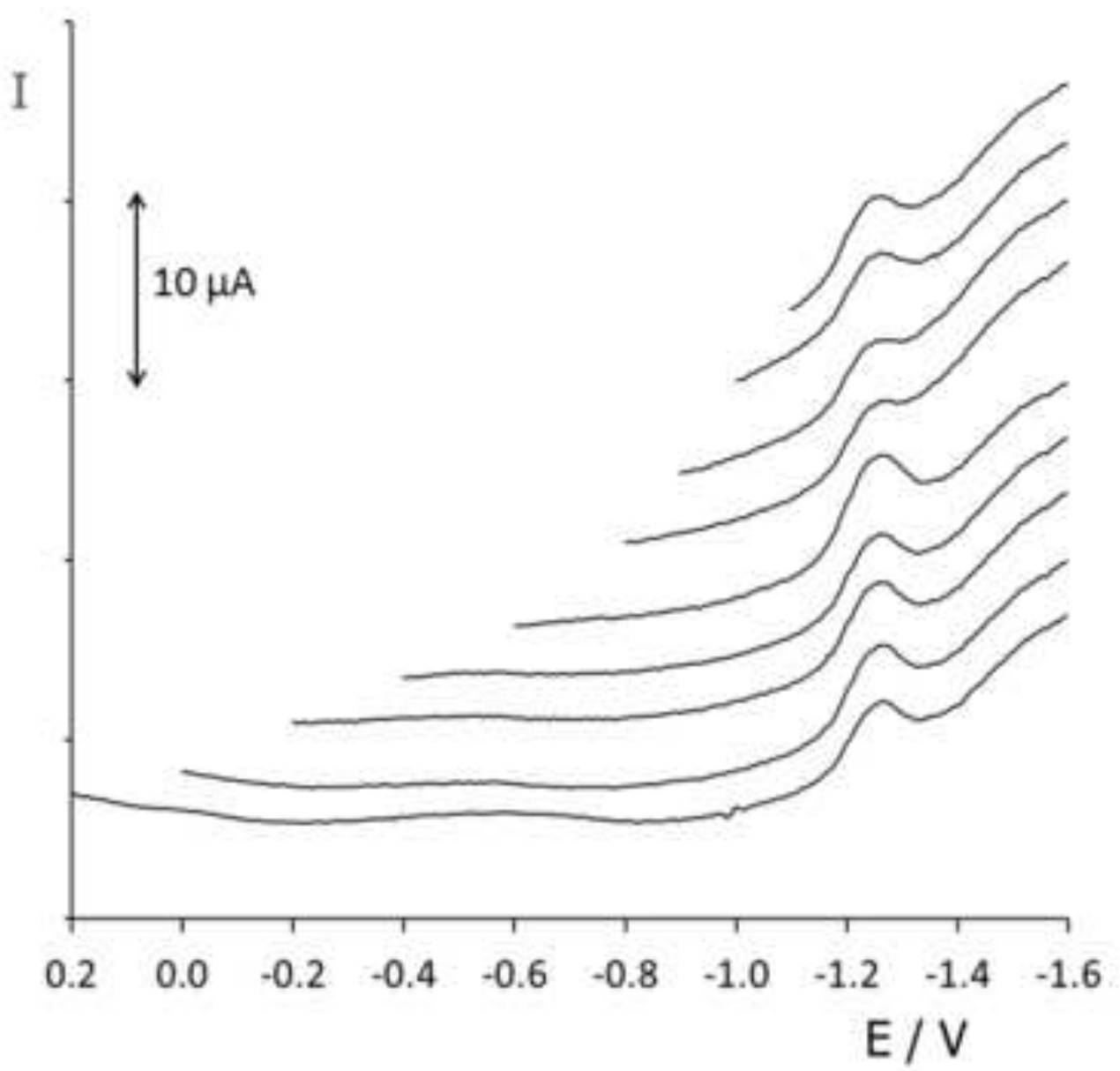


Click here to download high resolution image

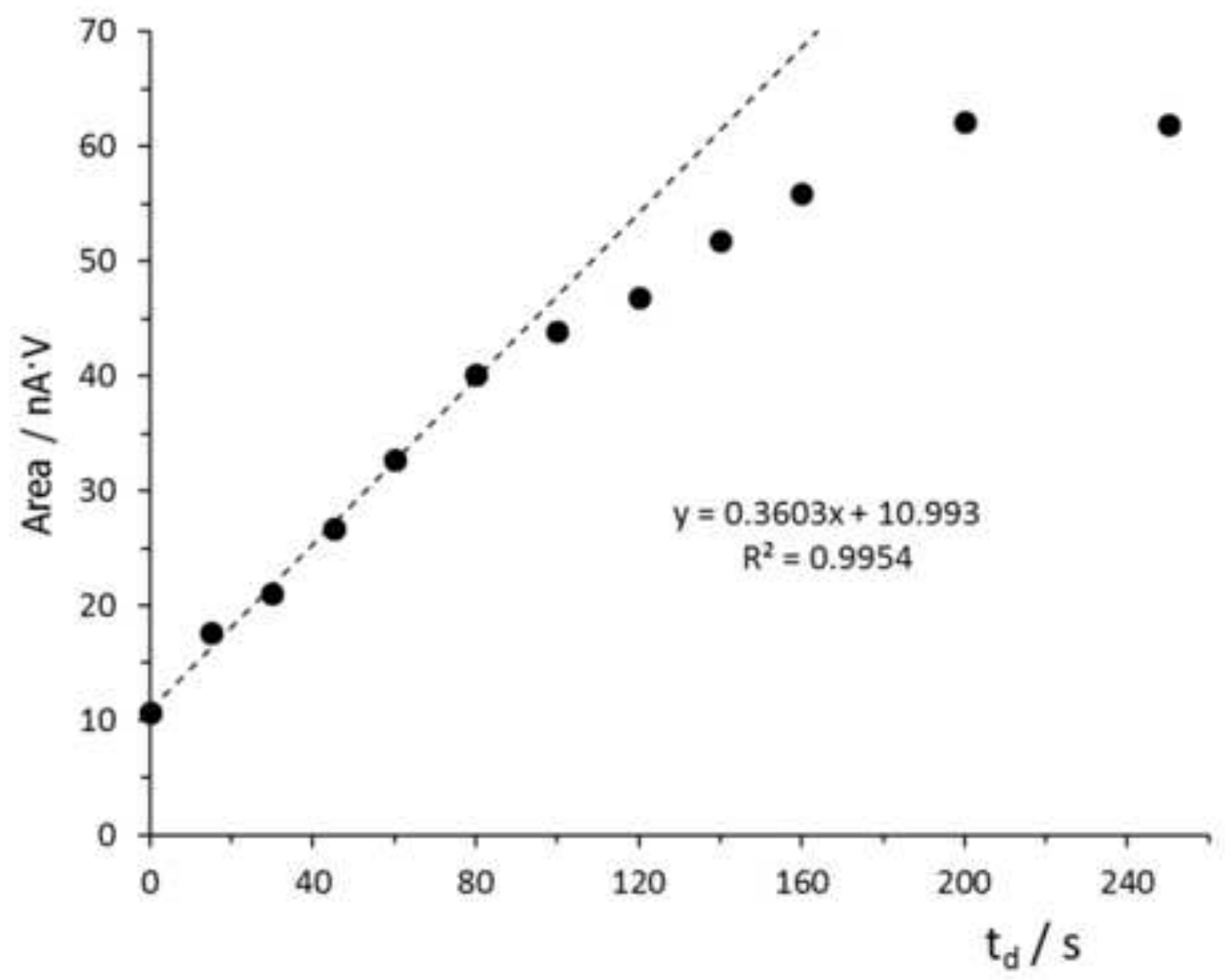




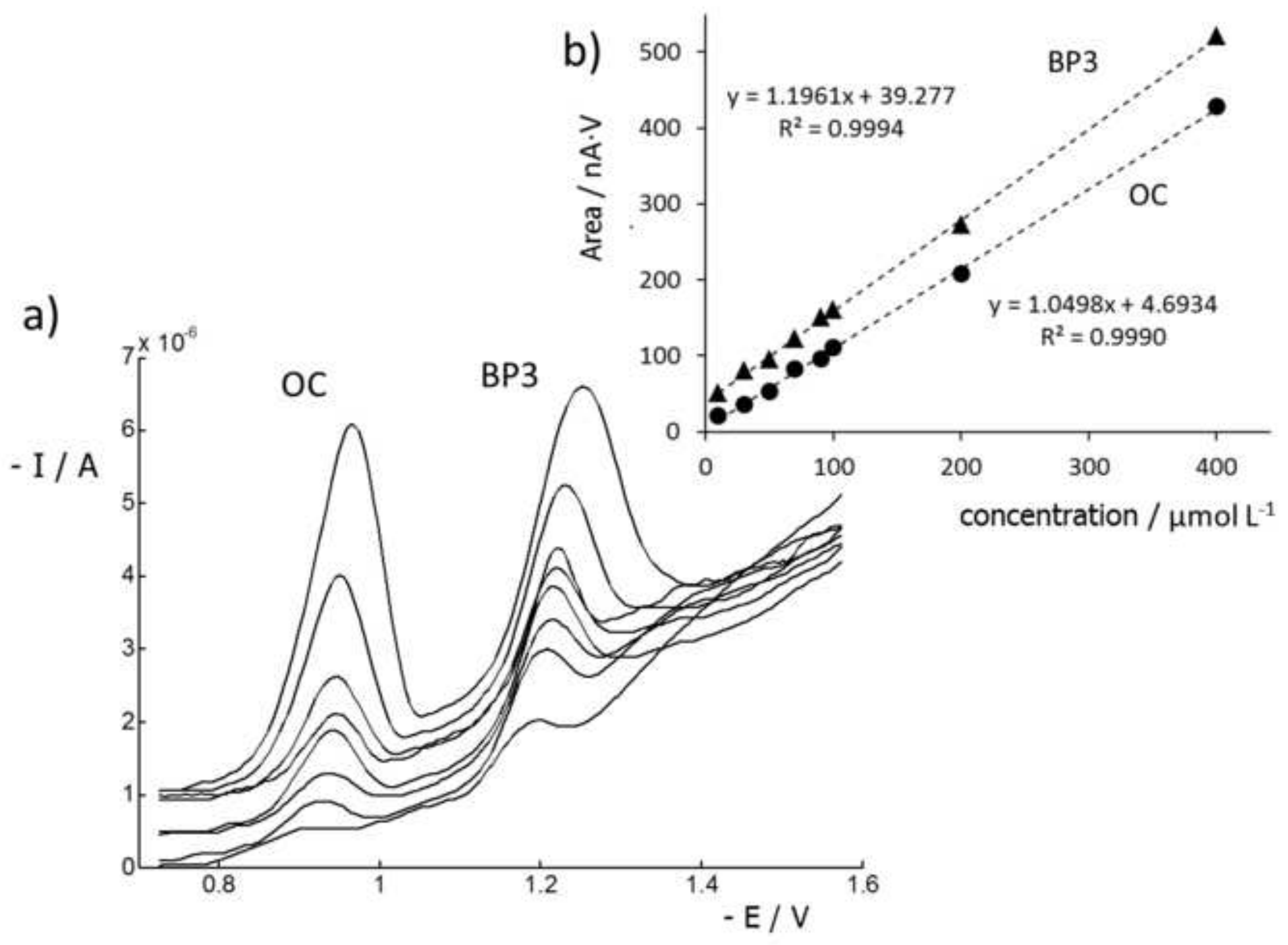




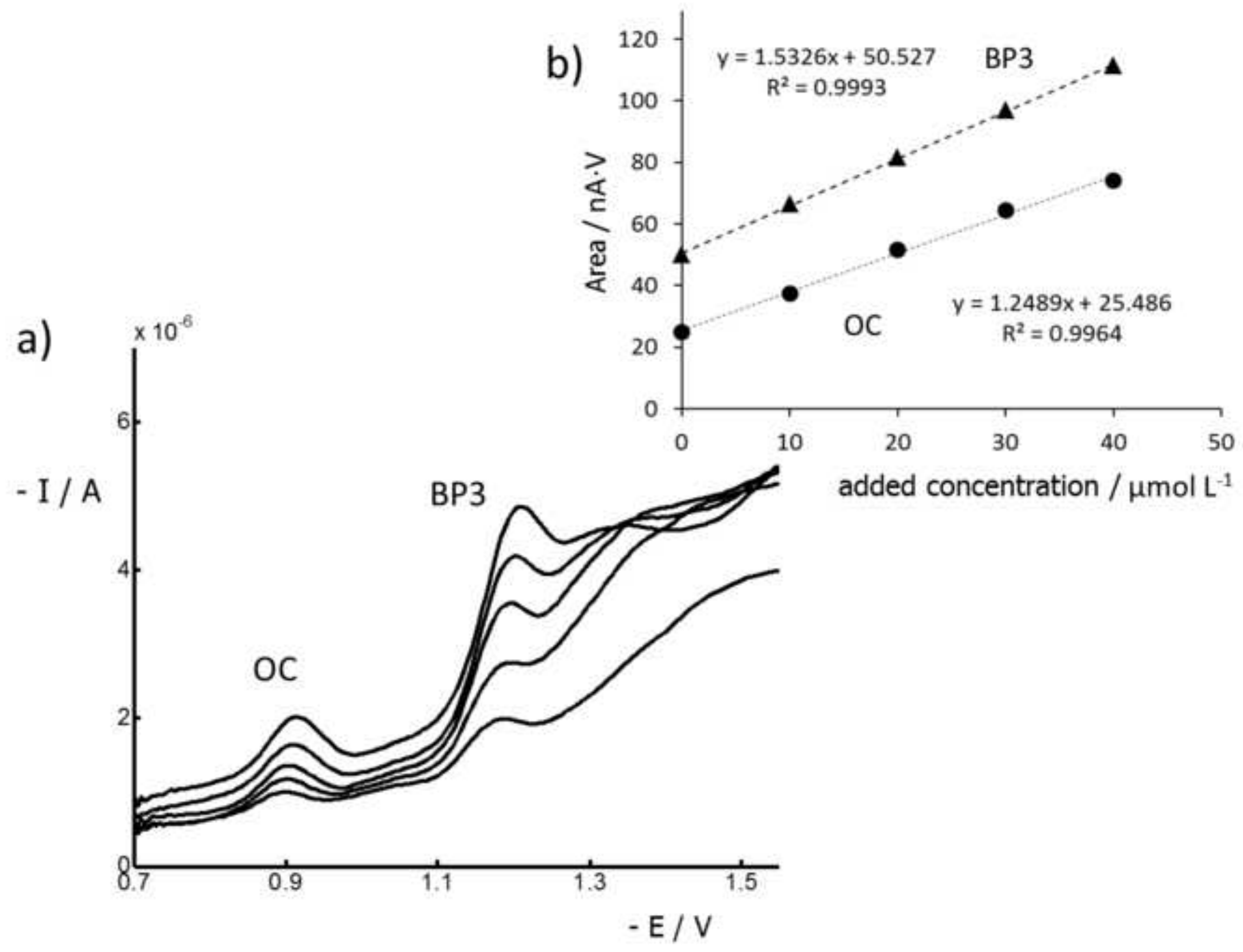




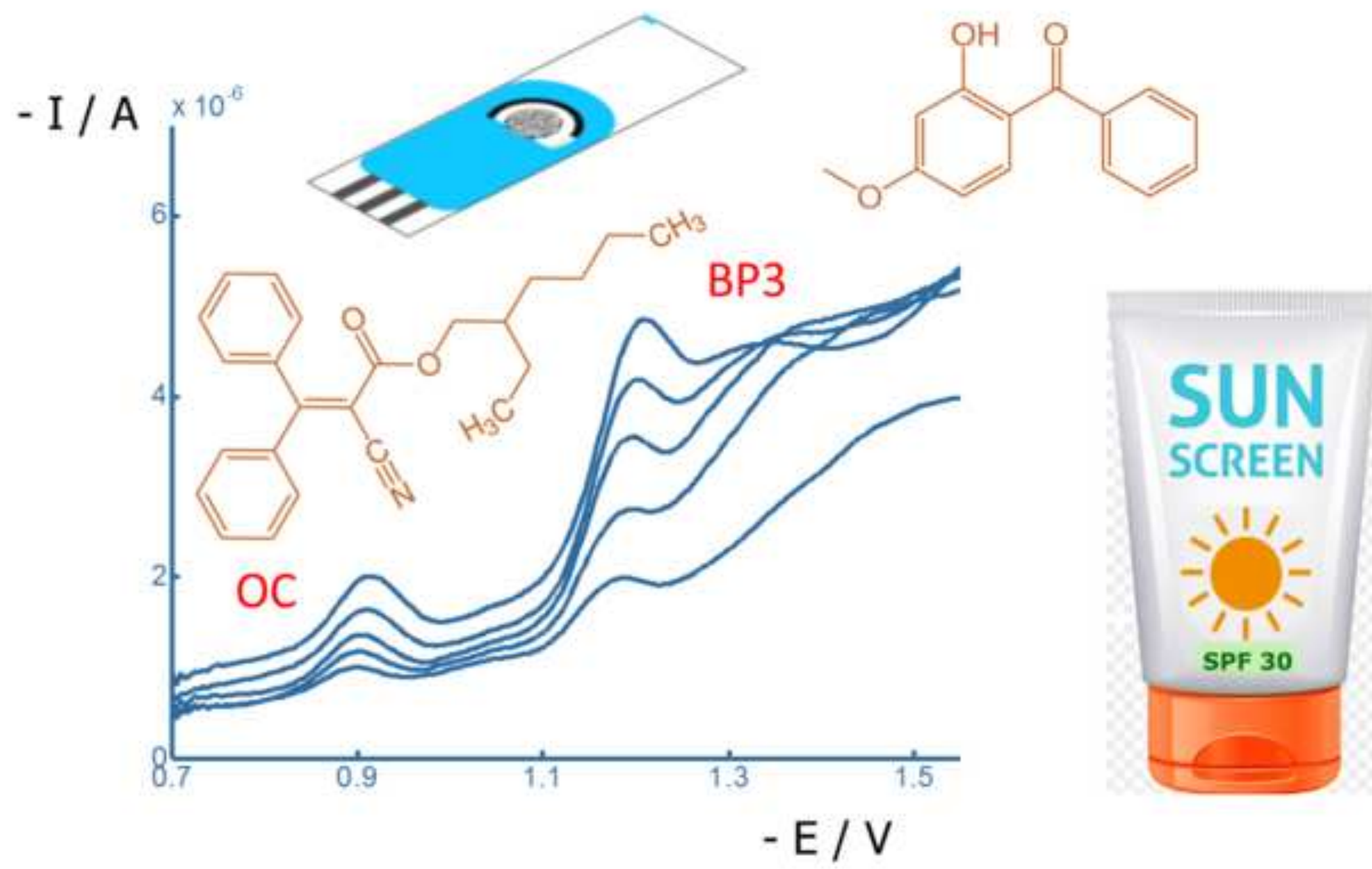

